Nach eingehender Würdigung der in der Literatur über diese seltene Frscheinung bestehenden Ansichten berichtet Thim müber einen Fall hämorrhagischer Hauterkrankung im Frühstadium der Lues. Der Pat. bekam etwa 4 Wochen nach Auftreten des Primäraffektes ein großpapulöses Exanthem, während das Allgemeinbefinden sich sehr verschlechterte: Schwäche, heftige Kopf- und Gelenkschmerzen, Fieber, Vergröblerung der Milz. 2 Tage später erschienen grellrote, runde, etwa 5markstückgroße, etwas erhabene Flecke, deren Zentrum sich allmählich dunkler färbte und binnen 3 Tagen zu einer mit dunklem, flüssigem Blute gefüllten Blase gestaltete. Der Grund einiger geplatzten Blasen erschien schlaff, dunkelrot, sebr empfindlich, bildete dann ein nur wenig Sekret absonderndes Geschwür und nahm nach Eintrocknung zur Kruste das Aussehen einer Rupia syphilitica an. Völlige Heilung erfolgte unter Injektionen von Sublimatkochsalzlösung neben warmen Soolbädern und lokalem Kalomelgebrauch. Verf. weist aus dem Krankheitsbilde nach, daß nicht etwa eine konizidierende Dermatose vorliege, sondern nur die syphilitische Erkrankung als Ursache der Hämorrhagien anzuschuldigen sei. Leider wollte der Pat. eine mikroskopische Untersuchung der Herde nicht zulassen. Verf. nimmt an, daß das syphilitische Virus die peripheren Gefäße geschädigt habe, wobei als disponierende Momente der elende Kräftezustand, schlechtes hygienisch-diätetisches Verhalten, ein längerer Aufenthalt in den Tropen und eine dort erw rbene Infektion mitsprechen könnten.

Max Joseph (Berlin).

Lebedew, A. Ein Fall von sogenanntem "Pemphigus syphiliticus adultorum". Journal russe de mal. cut. etc. 1901. Nr.8.

6 Jahre nach dem Primäraffekt entwickelten sich bei dem Patienten neben Papeln an den Handflächen und Fußsohlen auf entzündlich infiltriertem Grunde bis erbsengroße Blasen. Aus den Literaturangaben geht die große Seltenheit ähnlicher Fälle hervor. Auf eine spezifische Behandlung verschwinden die Blasen ohne Hinterlassung irgend welcher Folgen. Der sogenannte Pemphigus syphil, adultorum ist eine Modifikation eines papulösen - ein papulo-vesikulöses Syphilid.

Richard Fischel (Bad Hall).

Spitzer, Ludwig. Über Carcinombildung auf gummösem Boden. (Zeitschr. f. Heilk. Bd. XXIII. Heft 9. pag. 227).

Die Nenbildungen, die auf bestehenden luetischen Prozessen sich entwickeln, sind von jenen zu trennen. die auf Narben nach solchen sich bilden. Spitzer beschreibt, nachdem er eine Übersicbt über die einschlägige Literatur gegeben hat, einen in die erste Gruppe gehörigen Fall. Es handelte sich um eine gummöse Lues, die zu Ulzerationen an der Oberlippe geführt hatte, während gleichzeitig ein ausgebreiteter Defekt der knöchernen und häutigen Nase vorhanden war. Die außrordentliche Hartnäckigkeit des Ulzerationsprozesses einerseits, einzelne Charalktere des Geschwürs andererseits, ließen die Diagnose zwischen Carcinom auf Tuberkulose oder Carcinom anf dem Boden mehrjähriger gummöser Prozesse schwanken. In einem vom äußeren Rande der Geschwürsfläche excidierten 
Gewebsstückchen, fanden sich keinerlei Anhaltspunkte für die tuberkulöse Natur des Prozesses, auch Tierversuche gaben diesbezüglich ein völlig negatives Resultat, dagegen sah man in den Schnitten typische Carcinomnester. War damit die Diagnose Tuberkulose gefallen, so ging aus der nach Exstirpation der erkrankten Oberlippe vorgenommenen histologischen Untersuchung derselben mit noch größerer Wahrscheinlichkeit hervor, $\mathrm{da} B$ es sich um Carcinombildung auf gummöser Grundlage handelte. An den schon klinisch für Carcinom verdächtigen Stellen fand sich nämlich charakteristische atypische Epithelwucherung, während sich an den nicht carcinomatös veränderten Partien hauptsächlich Veränderungen der kleineren Gefäße vorfanden, die die Diagnose Lues zu stützen im stande waren, nämlich teils der Adventitia, teils der Muskelschichte angehörende, umschriebene knotenförmige Infiltrate mit beträchtlicher, oft bis zum totalen Verschluß des Lumens führenden Wucherung der Intima. Für die Diagnose Lues sprach auferdem in erster Linie die mehrjährige klinische Beobachtung des Falles. Alfred Kraus (Prag).

\section{Viscerale Syphilis.}

Rosenthal, 0. Über Erkrankungen des Herzens im Verla uf der Syphilis und der Gonorrhoe. Berl. klin. Wochenschrift 1900. Nr. 47, 48.

Rosenthal weist zunächst auf die auch heute nicht ganz überwundene Ansicht hin, welche Herzaffektionen syphilitischer Natur auf Quecksilbergebrauch zurückführt. Es folgen dann einige Literaturangaben, denen $R$ ef. die inzwischen erschienene Arbeit von $R u n$ eberg anreiben möchte. Nach Rosenthal treten die syphilitischen Herzaffektionen nicht wie allgemein angenommen 6-10 Jahre, sondern nach seinen Beobachtungen schon 2-4 Jahre nach der Infektion auf. Die gleichzeitig mit dem Ausbrauch des Exanthems auftretenden Herzpalpitationen u. s. w. sind als funktionelle Erscheinungen aufzufassen, die jeder organischen Beweis entbehren. Als Grund für diese Symptome glaubt Verf. die schlechte Ernährung des Herzens und die Wirkung des Syphilisgiftes auf Endo- und Myocard und die Herzganglien annehmen zu dürfen. Auf antisyphilitische Behandlung schwinden, wie auch ein Fall Rosenthals beweist, alle diese Symptome. In den Spätstadien ist es besonders das Myocard, das von der Syphilis ergriffen wird, u. zw. wie bei anderen Organen in Form der gummösen oder interstitiell fibrösen Erkrankung.

Verf. geht dann auf die detailiertere Schilderung der beiden Formen ein; er bezeichnet $\mathfrak{u}$. a. als sehr selten die syphilitische Affektion des Endocard, ebenso wie die des Herzbeutels, die meist vom Muskel her fortgeleitet sind. Es folgt dann ein kurzer Hinweis auf die isolierte Erkrankung der Coronararterien und dann auf die von Hallopea als angina pectoris syphilitica bezeichnete Herzaffektion. Unter den vielen 
schwärhenden Momenten, die zu einer syphilitischen Herzaffektion führen können, weist Verf. besonders auf die zeitweise Überanstrengung des Herzens, schwere körperliche Arbeit hin und auf den Mangel spezifischer Behandlung. Den Verlauf der Affektion bezeichnete Verf. im Gegensatz zu anderen als einen schleichenden. Die Prognose richtet sich je nach dem Stadium, in dem die Affektion zur Behandlung kommt.

Die gonorrhoische Herzaffektion befällt im Gegensatz zur syphilitischen meist das Endocard. Sie tritt gewöhnlich in der 4-6. Woche p. inf. auf, wenn der Prozeß in der Urethra auf deren bintere Teile übergegriffen hat. Von hier aus erfolgt der Übertritt der Gonokokken in die Blutbahn, in die dann eventuell auch gleichzeitig andere Mikroorganismen eindringen können. Die am häufigsten befallenen Partiea des Herzens sind die Aorten und Mitralklappen, das Pericard wird nur sehr selten betroffen. Klinisch tritt außer den physikalischen Phänomenen eine ausgesprochene Caebeseie in den Vordergrund, die sich oft mit einem der Pyo-Sephtaemie ähnlichen Bild vergesellschaftet, das nach Lesser in dem Absterben und Wiedereindringen der Gonokokken in die Blutbahn seine Ursache hat. So muB denn die Therapie die Krankbeitserreger zu zerstören trachten, weshalb Verf. die Behandlung der gleichzeitig bestehenden Urethritis befürwortet. Die Prognose ist immer unbestimmt; die Diagnose ist, auch wenn der Gonokokkennachweis nicht gelingt, aus dem ganzen Symptomenkomplex und dem Verlauf zu stellen.

Theodor Sachs (Frankfurt a. M.).

Runeberg, J. W. Die syphilitischea Herzaffektionen. Dtsch. med. Wochenschr. Nr. 1 u. 2. 1. und 8. Jan, 1903.

In einem historischen Berichte weist $\mathrm{R} u \mathrm{neberg}$ nach, wie lange man sich gesträubt habe, die Lues als ursächliches Moment für Herzaffektionen anzusehen. Erst mit dem Bekanntwerden der syphilitischen Affektionen in den Arterienwänden trat auch die Lehre von der Herzsyphilis in ein neues Stadium. Allerdings sei es auch bei genarer Kenntnis des betreffenden Falles stets schwer zu entscheiden, ob die Herzerkrankeng zweifellos syphilitischen oder anderen Ursprungs sei. Die beachtenswertesten Symptome bei Herzsyphilis seien "zeitweise oder auffallsweise vorkommende anginöse Schmerzen, cardiales Asthma, ungleiche und unregelmäßige Herzkontraktionen mit klanglosen Herztöilen und nicht distinkten Pulsschlägen, bisweilen wenn die Aortamündung oder die Klappen in den Prozej mit einbezogen sind, auch mit Nebengeräuschen, in einigen Fällen schließlich neben diesen Symptomen eine zunehmende anhaltende Herzinsuffizienz, verbunden mit Hypertrophie und Dilatation des Herzens". Lebensalter und Anamnese, sowie das Fehlen anderer Krankheitsursachen seien daneben zu berücksichtigen. Die häufigsten Formen der Herzsyphilis seien: Die sklerogummöse Arteriitis der Coronararterien mit nachfolgender Mymolacie und bindegewebigen Schwielen. Eigenartig ist hiebei der unverändert rubige Gesichtsausdruck. Sklerogummöse Aortitis und deren Folgen: Aortenklappenfehler, aneurysmatische Erweiterungen der Aorta, Herzhypertrophie. 
Seltener sind umschriebene Gummata im Herzmuskel, obgleich auch diese bereits in allen Teilen des Herzens beobachtet wurden. Etwas häufiger ist diffuse gummöte Myocarditis, zuweilen mit Coronararteriitis und Bindegewebsschwielen verbunden, nur in geringer Zahl warden gummöse Pericarditis und Endocarditis beobachtet. Statistische Daten ergaben eine fast ebenso große Mortalität bei Herzsyphilis als wie bei Lues der Nervenzentren. Nie solle đer Arzt versäumen in zweifelhaften Fällen auf Herzsyphilis zu fahnden, da in nicht allzu vorgeschrittenem Stadium oft völlige Heilung durch eine vorsichtige spezifische Behandlung zu erzielen sei. Max Joseph (Berlin).

Heine, L. Beitrag zurCasuistikder Mesaortitisgum mosa. (Virch. Arch. Bd. CLXII. Heft 2. pag. 257).

Es werden die Ergebnisse der Untersuchung von 3 Fällen von Aortenerkrankung mitgeteilt, bei denen die Autopsie anch sonst Zeichen florider Lues ergeben hat. Mikroskopisch fanden sich in der Media Entzündungsherde, die Leukocyten, Plasmazellen, epithelioide Zellen und Riesenzellen aufwiesen. In 2 der Fälle ergab sich keine Spur einer tuberkulösen Erkrankung, dagegen waren in allen Zeichen konstitutioneller Lues vorhanden. Dies spricht dem Verf. gegen die Annahme einer tuberkulösen Erkrankung. Auch führte die in 2 Fällen durchgeführte Untersuchung auf Tuberkelbazillen zu einem negativen Resultat. Endlich fand sich eine Endarterititis obliterans der vasa vasorum, was Verfasser gleichfalls für Lues zu sprechen scheint. Im Gegensatze hierzu fand Heine mehrfach mesaortitische Herde in Fällen, in denen Lues nicht vorlag. Im mikroskopischen Bilde fehlten dann die Riesenzellen. In zweifelbaften Fällen wird daher die Entscheidung über die Ätiologie der Aortenerkrankung Schwierigkeiten verursachen. Sind nurmebr Narben nach abgelaufenen Entzündungsprozessen vorhanden, so wird eine sichere Diagnose überhaupt unmöglich sein. Alfred Kraus (Prag).

Erdheim, J. Wien. (Abteilung Lang.) Nierengumma. Wiener medizin. Wochenschr. 1902. Nr. 10, 11 u. 12.

Erdheim teilt zuerst in extenso die Krankengeschichte eines Falles von Nierengumma bei einem 40jähr. Musiker mit. Derselbe war 7 Jahre zuvor intiziert gewesen. 3 Jahre nach der Infektion traten Gummen im Gesichte, über dem Orbitalrande, später Geschwüre am weichen Gaumen und Nase auf. Im weiteren Verlaufe trat eine ausgedebntere syphil. Nekrose des Stirnbeins und mehrfache Knochen- und Gelenkaffektionen auf. Plötzlich traten ohne Fieber oder sonstiges Unbehagen eine Hämaturie auf, im Sediment Blut und andere Zylinder, unter vielfachen Schwankungen wurde der Harn nach 2 Monaten normal. Die Hämaturie wurde auf ein Nierengumma bezogen, das ins Nierenbecken durchgebrochen ist, die Heilung erfolgte unter antilnetischer Behandlung in 2 Monaten. Für die Diaguose sprach der verhältnismäßig günstige Allgereinzustand, Fehlen von Fieber. in Sedimente bedeutende Schwankuagen des Blutgehaltes und Verschwinden der Nierensymptome auf antiluetische Behandlung, sowie die Antezedentien; neben dem Gumma 
konnte mit Wahrscheinlichkeit eine gleichzeitige Nephritis angenommen werden. Im Anschluße daran bespricht $\mathrm{Erdh}$ eim die ganze diesbezügliche Literatur in gründlicher und kritischer Weise.

Viktor Bandler (Prag).

\section{Syphilis des Nervensystems und der Sinnesorgane.}

Erb. "Bemerkungen zur pathologischen Anatomie der Syphilis des zentralen Nervensystems," Deutsche Zeitschrift für Nervenheilkunde. Bd. XXII. Heft 1 u. 2. p. $100 \mathrm{ff.}$

Ausgehend von eigenen und in der Literatur niedergelegten Beobachtungen pathologisch-anatomischer Befunde, unterzieht Erb die bisherigen Anschauungen über die Beziehungen zwischen Syphilis und cerebrospinalen Erkrankungen einer kritischen Betrachtung. Er teilt die auch von anderen Autoren ausgesprochene Ansicht, daß die anatomischen Verhältnisse allein wegen ihrer Mannigfaltigkeit sehr oft nicht zur Sicherung der Diagnose genügen, zumal die Ähnlichkeit, mit anderen pathologischen Prozessen ('Tuberkulose, Alkoholismus etc.) mitunter die Deutung des Befundes erschwert. Anamnese, klinischer Verlauf und der Errfolg der Behandlung seien für die Erkennung stets bedeutungsvolle Faktoren. Indem Erb seine Betrachtungen auch auf die scheinbar indifferenten Befunde von "nicht spezifischen" Degenerationsprozessen (z. B. bei Tabes und anderen cerebrospinalen Erkrankungen) ausdehnt, sucht er nachzuweisen, daß auch diese oft mit gleichem Rechte auf Syphilis zurückgeführt werden können, wie die sogenannten „spezifischen" gummösen Läsionen des Nervensystems und seiner Blutgefäße. Auch andere Ursachen könnten freilich zu solchen herd- oder strangförmigen Degenerationszuständen führen; jedenfalls müsse oft die Syphilis als Ur sache angenommen werden, trotz der bisher so erheblich erscheinenden Verschiedenartigkeit der "spezifischen" und "nicht spezifischen" Gewebsveränderungen. Den Begriff der „Parasyphilis" hält Erb für unhaltbar angesichts der Befunde von gleichzeitig bestehenden syphilitischen und para-(post-)syphilitischen Prozessen, sowie deren bisweilen beobachtetem Auftreten kurze Zeit nach der Ansteckung. In allen Einzelheiten der Arbeit, in welcher ein wertvolles kasuistisches Material gesichtet ist, muB auf das Original verwiesen werden. Fritz Callom on (Breslan).

Grosglick, A. und Weißbcrg, G. Akute Sehnervenentzündung als eine der ersten Erscheinungen sekundärer Lues. Monatshefte f. prakt. Dermatologie. Bd. XXXV.

Za. $4^{1 / 2}$ Monate nach dem infizierenden Coitus (der Primäraffekt war 2 Monate nach demselben aufgetreten) entwickelte sich bei dem Pat. das erste Exanthem, dessen Eruption durch einige Tage Gelenksschmerzen 
vorausgingen. Einige Tage nach Auftreten des Ausschlages Schmerzen im linken Auge, Sehschwäche beim Blick nach unten, die sich weiter so steigerte, daß Pat. nichts mehr sah; beim Blick nach oben Sehkraft sehr gering. Optkalmoskopischer Befund: Grenzen der linken Papille verwaschen, Papille wenig geschwollen, rot; Netzhautvenen erweitert. Unter allgemeiner spezifischer Behandlung Schwinden aller Erscheinungen. Sehkraft wie vor der Infektion. Nach Erörterung und Ausschluß aller Möglichkeiten, welche zur Neuritis optica hätten Veranlassung geben können, kommen die Verfasser zu dem Schlusse, daß es sich hier „um eine selbstständige akute spezifische Neuritis handelte, hervorgerufen durch primären Sitz des syphilitischen Prozesses im Sehnerven selbst"; besonders bemerkenswert ist das frühzeitige Auftreten.

Ludwig Waelsch (Prag).

Chmjelewsky, J. K. Zur Behandlung der syphilitischen Rückenmarkaffektionen am Odessaer Kujalnitzky-Liman Medicinskoje Obosrenje. Dezember 1901.

Auf Grund von 37 eigenen Beobachtungen kommt $\mathrm{Chmjelewsky}$ zum Resultat, daß bei luetischen Rückenmarksaffektionen, die auf eine spezifische Behandlung früher gut reagiert hatten, die Salz- und Schlammbäder vom Kujalnitzky-Liman bestens sich bewähren; aussichtslos sind bloß diejenigen $F a ̈ l l e$, in denen schon hochgradige anatomische Veränderungen eingetreten sind, speziell Erweichungsherde und sekundäre Degenerationen der Pyramidenbahnen. Die besten Resultate weisen die Bäder in den Fällen auf, in denen Hirnhäute und Nervenwurzeln Sitz der Affektion sind, also bei den sogenannten Randmyelitiden. Auf die chronischen, mehr schleichenden Krankheitsformen wirken die Bäder besser und nachhaltiger als auf die mehr akuten, in letzteren Fällen nur dann, wenn eine spezifische Behandlung vorher eine deutliche Besserung gebracht hat. Bei eintretender Besserung lassen zuerst die Sensibilitätsviel später erst die Motilitätsstörungen nach. Bei Meningo-Myelitis und Syphilis maligna praecox cerebro-spinalis sind Salzbäder von $27-29^{\circ} \mathrm{R}$. und Schlammbäder von $30-32^{\circ} \mathrm{R}$. in einer Zeitdauer von $15-30$ Minuten zu empfehlen. Mit einiger Vorsicht darf man bei syphilitischen Rückenmarksläsionen nach dem Typus Kahler, bei Erkrankungen des Konus, sowie bei Pseudo-tabes luetica Schlammbäder von $33-34^{0} \mathrm{R}$. in Anwendung bringen. Im offenen Liman dürfen die Patienten erst bei vollständiger Wiedergenesung baden, bloß den mit letztgenannten Affektionen Behafteten, sowie Personen mit chronischen Neuritiden ist es gestattet, schon im Frühstadium der Besserung von den Bädern im offenen Liman Gebrauch zu machen. Im allgemeinen jedoch sind letztere in der ersten Behandlungssaison nicht zu empfehlen, eher schon sind warme Seebäder von 10-15 Minuten Dauer am Platze.

Eine Kombination von Bädern mit Einreibungskur, resp. Jodpräparaten empfiehlt Verfasser nicht.

S. Pri $\beta \operatorname{mann}$ (Liban).

Patrick, Hugh T. The Somatic Ligns of Brain Syphilis. Journ. Amer. Med. Associat. XXXVII, 1100, Okt. 26. 1901. 
Patrick leitet seinen Vortrag mit den folgenden allgemeinen Bemerkungen ein: 1. Hirnsyphilis kommt am häufigsten vor in den ersten 3 Jahren nach der Infektion (zirka 50\%) und nimmt vom ersten Jahr an an Häufigkeit ab; nach dem 10. Jahre kommt sie nur ausnahmsweise vor.

2. Mangel einer Geschichte syphilit. Infektion ist für die Diagnose bei Frauen von gar keiner, bei Männern nur von untergeordneter Wichtigkeit.

3. Hirnsyphilis ist keineswegs identisch mit Hirngumma.

4. Am häufigsten liegt der Hirnsyphilis eine Arteristis zu Grunde, dann kommt syphil. Meningitis und Infiltration der Hirnnerven und zuletzt Gumma.

5. Lähmung infolge von Hirnsyphilis beruht meist auf Thrombose infolge von Arteriitis.

6. Daher sind viele Fälle von syphilit. Lähmung ebensowenig der Heilung zugänglich als Lähmungen nach gewöhnlicher Arteriosklerosis und Atherom.

7. Syphilis tritt nie als Systemerkrankung auf.

Die wesentlichsten Eigentümlichkeit der Hirnsypbilis besteht in der Abwesenheit irgend eines regelmäßjgen Typus. Kopfschmerz ist in zirka $75 \%$ aller Fälle vorhanden, anfangs nicht dauernd, später fortwährend, nicht immer Nachts vorhanden. Die Lokalisation ist nicht von Bedeutung, Druckempfindlichkeit irgend einer Stelle am Kopf ist nicht immer nachzuweisen. Vorübergehende Anfälle von Schwindel, Synkope, Bewußtlosigkeit, lokalisierte Sensationen, Gefühl von Taubheit oder Prickeln, Krämpfe kommen häufig vor, nam. epileptiforme. Von großer Wichtigkeit sind Lähmungen der Hirnnerven, namentlich der Augennerven, ebenso Störungen des Gesichtsfeldes. Weniger häufiger kommen Lähmungen des 5. und 7. Nerven, aber auch die unter dem 8. gelegenen Nerven sind nicht ganz verschont. Besondere Aufmerksamkeit wird gerichtet auf die gleichzeitige Lähmung verschiedener nicht benachbarter Nerven. Übergreifen von Meningitis auf die Rückenmarkshäute kann gleichzeitig spinale Symptome hervorrufen. Fingehender werden die einzelnen Erscheinungen der drohenden oder vollendeten Thrombose in ihren mannigfaltigen Bildern besprochen. Ferner wird auf einen eigentümlichen Stupor mancher an Hirnsyphilis Erkrankter hingewiesen. Fieber ist selten außer bei Erkrankung des Pons und der medull. oblongat. Erbrechen ist kein regelmäßiges Symptom, Polydipsie, Polyurie und Polyphagie sind nicht ungewöhnliche Folgen, ebenso Schlaflosigkeit.

H. G. Klotz (New-York).

Kopezynski (Berlin). Zur Kenntuis der Symptomatologie und pathologischen Anatomie der Lues Cerebri. Deutsche Zeitschrift für Nervenheilkunde 1901. Bd. XX, p. 216.

Im Anschluß an die genau ausgeführte mikroskopische Untersuchung eines Falles von Hemiplegia alternans superior bespricht $K$. eingehend die Gefäßveränderungen. Die starke Sekundärdegeration im Rückenmark 
war auf die Pyranidenseitenstrangbahnen beschränkt, während im Vorderstrang eine solche fehlte, sodaß totale Kreuzung anzunehmen ist.

Dewey, Richard. The Psychoses in Cerebral Syphilis. Journ. Americ. Med. Associat. XXXVII. 1102, Olst. 26, 1901.

Nach Dewey wird die Beurteilnng des Einflusses der Syphilis auf die Entstehung von Geisteskrankheiten dadurch erschwert, dab auf der einen Seite neben nachweislicher Syphilis andere Ursachen der Geistesstörung, wie Alkoholismus, Altersveränderungen etc. anwcsend sein können, andererseits Syphilis nicht absolut ausgescblossen werden kann, auch wenn eine Geschichte früherer Erkrankung oder der Nachweis anderer Syphiliserscheinungen nicht beigebracht werden können. Nach Besprechung der Ansichten verschiedener Autoren kommt er zu dem Schlusse, daß es zur Zeit verfrüht erscheine syphilitische Psychosen als Typen aufzustellen bei der Unbestimmtheit unserer Kenntnisse über die Beziehungen gewisser psychischer Symptome zu gewissen molekulären oder selbst gröberen Veränderungen im Hixn, bei dem Unvermögen uns eine Vorstellung zu machen von der Art und Weise, wie psychische Störungen durch pathologische Zustände zustande gebracht werden können, endlich bei der vorläufig doch nur versuchsweisen und auf Vermutungen basierten Klassifikation der Psychosen überhaupt. Auch seine persönlichen Erfahrungen geben D. keinen Anhalt für bestimmte Ansichten. Unter 1200 Fällen von allen möglichen Nerven- und Geisteskrankheiten fand er 45 gutbegründete Fälle von konstitutioneller Syphilis; darunter waren 17 von Paresis, 12 Hirnsyphilis mit Symptomen organischer Veränderungen; 7 waren Psychosen ohne Symptome syphilitischer Veränderungen, $4 \mathrm{De}$ mentia, 4 zeigten Ophthalmoplegia und Neuritis optica, 4 hypochondrische Melancholie, 2 Tabes und 3 senile Formen. H. G. Klotz (New-York).

\section{Hereditäre Syphilis.}

Hostalrich, M. Du rôle de la syphilis héréditaire en tératologie. Thèse de Montpellier 1902.

Hostalrich ergeht sich in einer sehr lesenswerten ausführlichen Arbeit über die Rolle, welche die hereditäre Lues beim Zustandekommen von Mißbildungen und Dystrophien spielt und berichtet als Beitrag zur Kenntnis dieses Einflusses über eine Reihe von sehr interessanten, aus Toulon stammenden, bis jetzt nicht publizierten Beobachtungen.

Dieselben seien in aller Kürze hier angeführt:

1. Ein doppelköpfiger Foetus, zu rechter Zeit geboren, von einer syphilisfreien Frau, deren Mann 5 Jahre vor der Geburt des Monstrums syphilitische sekundäre Symptome geboten hatte. Die zwei Gesichter waren sehr gut ausgebildet, das knöcherne Schädeldach fehlte vollkommen. 
2. Ein anencephaler Foetus, geboren am normalen Schwangerschaftsende von einer Frau, welche 4 Jabre vorher eine Genitalsklerose gehabt hatte.

3. Ein anencephaler syphilitischer Foetus, von einer Primipara stammend, welche wahrscheinlich auch Syphilis hatte. Dieselbe Frau gebar 2 Jahre später abermals einen anencephalus.

4. 8monatlicher hereditär-syphilitischer Foetus, welcher mit einer Nabelhernie geboren wurde, die den größten Teil der Baucheingeweide beherbergte. Die Mutter war $6 \mathrm{mal}$ gravid, darunter 3 Todgeburten.

5. Neugeborenes Kind am normalen Schwangerschaftsende geboren mit angeborener linksseitiger Oberschenkelamputation. Die Mutter hatte vier Jahre vorher einen indurierten Schanker am Genitale. stammend.

6. Foetus mit Hasenscharte, von einem syphilitischen Vater her-

7. Hemimelische Todgeburt, deren Obduktion eine voluminöse Leber, eine vergrößerte Milz und typische syphilitische Knochenveränderungen zeigte.

8. Neugeborenes Kind einer manifest syphilitischen Frau mit Mißbildungen der Ohrmuschel behaftet.

9. Hydrocephalischer Foetus einer eingestandenermaßen syphilitischen Mutter, welcher einige Minuten nach der Geburt verstarb.

10. Frucht mit Syndaktylie und löffelförmiger Verbildung der linken Hand. Die Mutter war eine syphilitische Publica.

Hochsinger (Wien).

Scherer, Franz. Die Parrotschen Pseudoparalysen bei angeborener Syphilis. Jahrb. f. Kinderheilk. Bd. LV. Heft 5 .

Scherer nnterzieht die verschiedenen Anschauungen über die Genese der Extremitäten-Lähmungen bei kongenital-luetischen Säuglingen zunächst einer ausführlichen Betrachtung und neigt zu der Anschaung, daß die Parrotsche Lehre von der ossalen Genese der Extremitätenlähmungen bei hereditärer Syphilis nicht ansreicht, speziell dessen Lehre, daß es sich um eine bloße Ruhigstellung infolge der schmerzhaften Knochenerkrankung handelt, trifft nur in seltenen Fällen zu. Scherer selbst fand unter 11 Fällen von Extremitätenlähmungen nur in 4 Fällen die Wegnerschen Knochenveränderungen; andererseits fand er in 8 Fällen von hereditärer Syphilis Weg $n$ ersche Knochenveränderungen ohne Lähmung.

Angeregt durch die Befunde von Schlichter und $\mathrm{Z}$ appert über anatomische Veränderungen im Rückenmark bei hereditärer Syphilis (welche nach Ansicht des Referenten Irrtümer sind), nahm Scherer histologische Untersuchungen des Zentralnervensystems bei seinen Fällen vor, welche aber weder mit Hilfe der $\mathrm{N}$ isselschen noch mit Hilfe der Marchischen Methode pathologische Veränderungen aufdeckten. Hin* gegen fanden sich sowohl im Rückenmark, wie in den übrigen Organen des Körpers vielfache Streptokokkenembolien. 
In diesen Befunden erblickt nun $\mathbf{S}$ cherer den Ausdruck einer zur Lues hinzugetretenen sekundären septischen Infektion und erklärt die Lähmung als toxische Einwirkung entweder des luetischen Toxins oder der im Blute kreisenden septischen Mikroben.

$$
\text { Hochsinger (Wien). }
$$

de Rothschild, Henry. Contribution à l'étude de la pseudosyphilis. Revue d'hygiène et de médecine infantiles. Tome I. Nr. 2.1902.

de Rothschild weist darauf hin, daß nicht allein die Syphilis, sondern auch chronische Darmerkrankungen bei künstlich genährten Neugeborenen und Säuglingen $z u$ schweren Kachexien führen können, welche zu denselben klinischen Bildern führen können, wie hereditäre Syphilis und warnt davor, aus bloßer Kachexie ohne sonstige manifeste Symptome hereditäre Lues zu diagnostizieren.

Für solche syphilisähnliche Sänglings-Kachexien den Ausdruck „Pseudosyphilis" zu gebrauchen, ist nach Ansicht des Referenten überflüssig. Hochsinger (Wien).

Fournier, Edmond. Des dystrophies veineuses de l'hérédosyphilis. Revue d'hygiène et de médecine infantiles Tome I. Nr. 1.

C. Hochsinger. A propos des dystrophies veineuses de l'hérédosyphilis. Ibidem Nr. 2.

E. Fournier. Replik ibidem.

E. Fournier beschreibt unter dem Titel „Des dystrophies veineuses de l'hérédosyphilis" das Vorkommen von Erweiterungen der subkutanen Schädelvenen, welche als dicke blauschwarze oder blaxviolette Stränge durch die Haut durchschimmern. Im Säuglingsalter ist die Störung auf das Gebiet der V. jugularis externa beschränkt, wie durch ausgezeichnete photographische Reproduktionen und durch genaue anatomische Schilderungen der Verästlungsgebiete der subkutanen Schädelvenen dargelegt wird.

E. Fournier berichtet, daß er an allen 30 Kindern, welche mit dieser Dystrophie der Venen behaftet waren, Zeichen von hereditärer Lues auffinden konnte, und daß, mit Ausnahme eines einzigen Falles, alle diese Kinder von syphilitischen Ascendenten abstammten. Zur Erklärung dieser Venen-Ektasien nimmt Fournier an, daß bei Descendenten syphilitischer Eltern eine angeborene Schwäche des Venensystems besteht. Die im frühen Kindesalter zu Staunng in der oberen Hohlvene prädisponierenden mechanischen Verhältnisse (Säugen und Schreien) bilden eine dauernde Schädigung des Venensystems, deren sich dieses infolge der angeborenen Schwäche nicht erwehren kann. Im späteren Kindesalter bilden sich die Venenerweiterungen wieder spontan zurück. Auch wird ein von Parrot stammendes Präparat abgebildet, bei welchem (es handelte sich um ein hereditär syphilitisches Kind) eine enorme Ausdehnung der subkutanen Venen des Thorax und des Abdomens, sowie der unteren Extremitäten der rechten Seite bestand. Leider ist der Sektionsbefund dieses Kindes nicht bekannt geworden. Die Abbildung bei Fournier ist nach einer Moulage, welche Parrot anfertigen lieB, 
erfolgt. Nach dem Bilde zu schließen, dürfte es sich wohl um ein Zirkulationshindernis in der Pfortader, vielleicht infolge syphilitischer Lebererkrankung handeln. (Ref.)

Hochsinger legt in einem ausfübrlichen Schreiben an die Redaktion der Revue dar, daß die von Fournier beschriebenen Venenerweiterungen mit der hereditären Syphilis in keiner Beziehung stehen. Er gibt des weiteren an, dafo er die Ektasien der Schädelvenen bei schwerer Schädelrachitis und bei Hydrocephalus gefunden hat, kurz unter Verhältnissen sah, bei welchen ein erhöhter Binnendruck in der Schädelkapsel bestand. Dieser ist für das Hervortreten der subkutanen Schädel. venen einzig und allein verantwortlich zu machen. Weil nun Schädelrachitis und Hydrocephalus zu den häufigen Begleitern der hereditären Frühsyphilis gehören, findet man in der Tat die von diesen Zuständen abhängigen Venenausdehnungen am Schädel bei syphilitischen Säuglingen sehr häufig.

In seiner Replik meint Fournier, daß Hochsingers Rekriminationen seine Lehre bestätigen, indem Hochsinger ansdrücklich feststellt, daß Schädelrachitis und Hydrocephalus zu den treuesten Begleiterscheinungen der hereditären Frühsyphilis gehören.

$$
\text { Hochsinger (Wien). }
$$

Peters, R. Über Erkrankungen des Rückenmarks bei hereditär-syphilitischen Neugeborenen und Säuglingen. Jahrb. f. Khk. 53, der dritten Folge. Bd. III. 3. Heft.

Peters stellt die Behauptung auf, die bisherige Anschaung, selbständige Affektionen des Rückenmarks kämen bei hereditär-syphilitischen Säuglingen nicht zustande, sei durch seine Beobachtungen unhaltbar geworden. Die lähmungsartigen Haltungen der Oberextremitäten, die bei syphilitischen Neugeborenen vorkommen, die Parrotsche Pseudoparalyse und die von Hochsinger geschilderten Faustbildungen bei hereditär-syphilitischen Säuglingen sind nach Peters Ansicht auf tiefere Veränderungen im Nervensystem, d, h. auf syphilitische Affektion des Rückenmarks zurückzuführen.

Wiewohl Peters von "gewöhnlichen syphilitischen makroskopisch nachweisbaren Gewebsalterationen, gumata etc." spricht, die in diesen, speziell Hochsingers Fällen die Grundlage für die orwähnten Erscheinungen abgeben sollen, hat er leider selbst - keine einzige derartige "makroskopisch nachweisbare" Rüchenmarksalteration wirklich gesehen. Er beschränkt sich vielmehr darauf, einen klinisch-symptomatischen Beweis (?) für seine Behauptung zu liefern.

Aus dem Symptomenbilde, das ihm 11 an derartigen Lähmungserscheinungen erkrankte hereditär-syphilitische Kinder darboten, spez. aus dem von ihm beschriebenen Symptom der "Flossenstellung“ der Hände (Herabhängen, Pronationsstellung bei ulnarer Abweichung der Hände) schließt er auf komplette Lähmung des unteren, oberen oder beider Abschnitte des betreffenden Cervicalteiles des Rückenmarks und zwar, wie wir gleich vorwegnehmen wollen - auf einen herdweise an- 


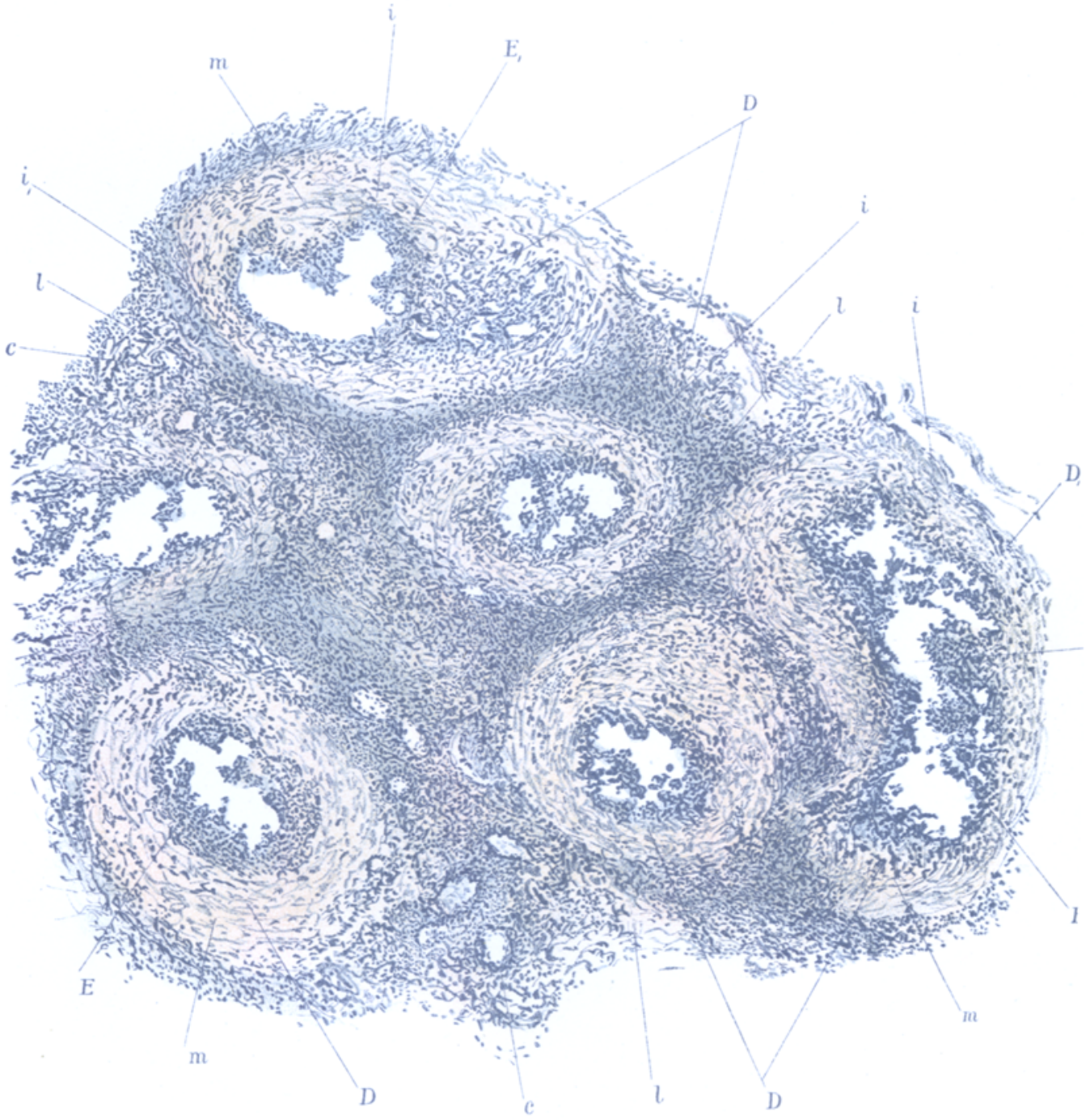

Nobl: Histologie der blennorrh. Deferenitis u. Epididvnitus 